\title{
For a better adjuvant strategy for resected lung cancer-lessons from treatment failure patterns of the ADJUVANT trial (CTONG 1104)
}

\author{
Kenichi Suda \\ Division of Thoracic Surgery, Department of Surgery, Kindai University Faculty of Medicine, Osaka-Sayama, Japan \\ Correspondence to: Kenichi Suda, MD, PhD. Division of Thoracic Surgery, Department of Surgery, Kindai University Faculty of Medicine, 377-2 \\ Ohno-Higashi, Osaka-Sayama 589-8511, Japan. Email: ascaris@surg2.med.kyushu-u.ac.jp. \\ Provenance: This is an invited article commissioned by the Academic Editor Dr. Xianglin Hu (Department of Pulmonary Medicine, Zhongshan \\ Hospital, Fudan University, Shanghai, China). \\ Comment on: Xu ST, Xi JJ, Zhong WZ, et al. The unique spatial-temporal treatment failure patterns of adjuvant gefitinib therapy: a post hoc analysis \\ of the ADJUVANT trial (CTONG 1104). J Thorac Oncol 2019;14:503-12.
}

Submitted Aug 10, 2019. Accepted for publication Aug 27, 2019.

doi: $10.21037 /$ tlcr.2019.08.24

View this article at: http://dx.doi.org/10.21037/tlcr.2019.08.24

Surgical resection is a mainstay treatment for most patients with early-stage non-small cell lung cancer (NSCLC). With the recent improvements in the 5 -year survival rates after lung cancer surgery (1), many thoracic surgeons are becoming interested in less invasive surgical techniques, such as sublobar resection for smaller NSCLC (2) or robotic (3) and uniportal (4) approaches to reduce the invasiveness of the chest wall. However, in patients with pathological stage II to III disease, the risk of post-surgical recurrence is still problematic even when complete locoregional control is thought to have been achieved. Currently, doublet chemotherapy [vinorelbine plus cisplatin (VP)] is usually administered as a standard adjuvant treatment for these patients (5); however, the clinical benefit is small, increasing the 5 -year survival rate by only $5.4 \%$ (6). In the advanced disease setting, molecular targeted therapies [tyrosine kinase inhibitors (TKIs)] and immune checkpoint inhibitors have shown higher efficacy and lower toxicity than doublet chemotherapies (7). This has encouraged the start of clinical trials using TKIs or immune checkpoint inhibitors in the adjuvant setting for patients with earlier-stage lung cancer (8).

Among several trials that enrolled patients with NSCLC with epidermal growth factor receptor $(E G F R)$ mutation to evaluate the roles of adjuvant EGFR-TKI monotherapy, the EVAN trial and the ADJUVANT trial are the first two studies that were performed to completion. The EVAN trial was a randomized phase II study that compared 2 years of erlotinib, a first-generation (1G) EGFR-TKI, with VP chemotherapy in patients with surgically resected stage IIIA NSCLC with EGFR mutation (9). The ADJUVANT trial was a randomized phase III study that compared 2 years of adjuvant gefitinib, another 1G EGFR-TKI, with VP chemotherapy in patients with surgically resected stage II to IIIA (N1-N2) NSCLC with EGFR mutation (10). Both trials demonstrated significantly longer diseasefree survival (DFS) in favor of adjuvant EGFR-TKI monotherapy compared with VP chemotherapy. However, whether adjuvant $1 \mathrm{G}$ EGFR-TKIs can achieve their "primary purpose" of adjuvant therapies (i.e., to increase the postoperative cure rate) remains unclear. According to the ADJUVANT trial, the DFS Kaplan-Meier curves exhibited clear separation at around 12 months, but then came together at around 36 months with no apparent tail of long-term disease-free survivors in either treatment arm. Similarly shaped DFS curves were observed in the subgroup analysis of patients with EGFR mutation-positive cancer in the phase III RADIANT trial, in which patients were allocated to 2 years of erlotinib or placebo either following completion of adjuvant chemotherapy or without adjuvant chemotherapy (11). While the results of the EVAN trial seem encouraging, an exploratory post hoc analysis of the EVAN trial showed a significantly better 3-year DFS in the erlotinib group than in the VP chemotherapy group [54.2\% vs. $19.8 \%$, respectively; relative risk, $2.735 ; 95 \%$ confidence 
interval (CI), 1.018-7.347; $\mathrm{P}=0.0460$ (9)]. However, it seems possible that both DFS curves will come together at around 4 years. These study findings may indicate that $1 \mathrm{G}$ EGFRTKI monotherapy, as an adjuvant therapy, is not able to eradicate all of the residual tumor cells. This is similar to the clinical experience of EGFR-TKI monotherapy for patients with advanced disease; almost all patients will develop acquired resistance to EGFR-TKI despite a dramatic initial response (12).

Then, how can we improve the cure rate in patients with surgically resected lung cancer with EGFR mutation by adjuvant therapy? A recent paper by Xu et al. (13), which reported on the spatial-temporal recurrence patterns in the ADJUVANT trial, may help to answer this question. In their paper, the temporal distribution analysis showed lower tumor recurrence with gefitinib than with VP chemotherapy 0 to 21 months after surgery. However, recurrence with gefitinib showed a constant rate of increase. The authors also performed analyses based on recurrence sites [central nervous system (CNS) metastases vs. extracranial metastases]; they observed a higher risk of CNS metastasis than extracranial metastasis in the gefitinib arm, while the risks of both types of metastasis seemed to be similar in the VP chemotherapy arm.

Before I further discuss how adjuvant gefitinib changes the hazard rate of recurrence and how to lower this hazard rate as a next step, I would like to briefly summarize the data about "natural" (i.e., without adjuvant therapy) recurrence patterns after lung cancer surgery. In a large cohort study, Demicheli et al. (14) retrospectively analyzed 1,506 patients who underwent surgical resection of NSCLCs (only $12 \%$ and $4 \%$ received adjuvant chemotherapy and adjuvant radiotherapy, respectively). In their analysis, an initial surge of any treatment failure occurred 9 months after surgery, followed by two smaller peaks at the end of the second and fourth years. In another retrospective study, Park et al. (15) reviewed the medical records of 333 patients with pathological N1 disease (103 did not receive adjuvant chemotherapy) and found that the hazard rate of recurrence peaked at around 9 and 24 months, and a third smaller peak occurred during the fourth year. The curve of the hazard rate of recurrence in patients who received adjuvant chemotherapy exhibited a more delayed first peak and lower hazard rate during the entire observation period. Based on these data, I modeled a curve of the hazard rate of recurrence in patients who had undergone surgical resection of NSCLC without adjuvant therapy (Figure 1A); I then modified this curve based on each adjuvant therapy.
Figure $1 B$ is a model that shows when patients received adjuvant VP chemotherapy. The objective response rate for platinum doublet chemotherapy in patients with advancedstage NSCLC is reportedly around 30\% (16). Therefore, the height of the curve of the hazard rate of recurrence was lowered by 0.7 times. However, it is anticipated that tumor cells, which are damaged by VP chemotherapy, will become dormant to survive (drug-tolerant cells) and that these drug-tolerant cells will then start to regrow after a certain period (the purple curve in Figure 1B, left). Combining these two curves produces an estimated curve of the hazard rate of recurrence in patients who receive VP adjuvant chemotherapy (Figure 1B, right), and this curve is similar to that described in the paper by Xu et al. (13). An estimated curve for the hazard rate of recurrence in patients who received adjuvant gefitinib [the objective response rate of which is $70 \%$ in patients with advanced disease $(17,18)]$ was also generated (Figure 1C). In this model, the hazard rate curve for regrowth of drug-tolerant cells is estimated to be higher and to show a continuous increase during the observation period because more patients in the gefitinib arm will possess drug-tolerant cells (because of the higher efficacy of gefitinib) and a longer duration of treatment (2 years in the ADJUVANT trial). The estimated curve of the hazard rate of recurrence is also similar to that described in the paper by $\mathrm{Xu}$ et al. (13).

Another remarkable finding in the study by $\mathrm{Xu}$ et al. (13) was the higher hazard rate of recurrence for CNS metastases than for extracranial metastases in the gefitinib arm, as described above. This can be explained by poorer blood-brain barrier (BBB) penetration by gefitinib because a comparison study of primary lung tumors and brain metastases showed that both tumors are genetically similar (19).

Considering these assumptions, how can we improve the outcomes of patients who have undergone surgical resection of EGFR mutation-positive lung cancer? First, a strategy to suppress the emergence of CNS recurrence is needed. As described above, the higher hazard rate of CNS recurrence than extracranial metastases in patients who received adjuvant gefitinib might have been due to poorer BBB penetration by gefitinib. Therefore, an EGFR-TKI with better BBB penetration, such as AZD3759 (20) or osimertinib (21), will provide better treatment outcomes as adjuvant EGFR-TKI therapy. Second, surviving drugtolerant cancer cells should be targeted because the impact of regrowth of drug-tolerant cells seems to be larger in patients who have received adjuvant EGFR-TKI (Figure $1 C$, left) than in those who have received VP chemotherapy 

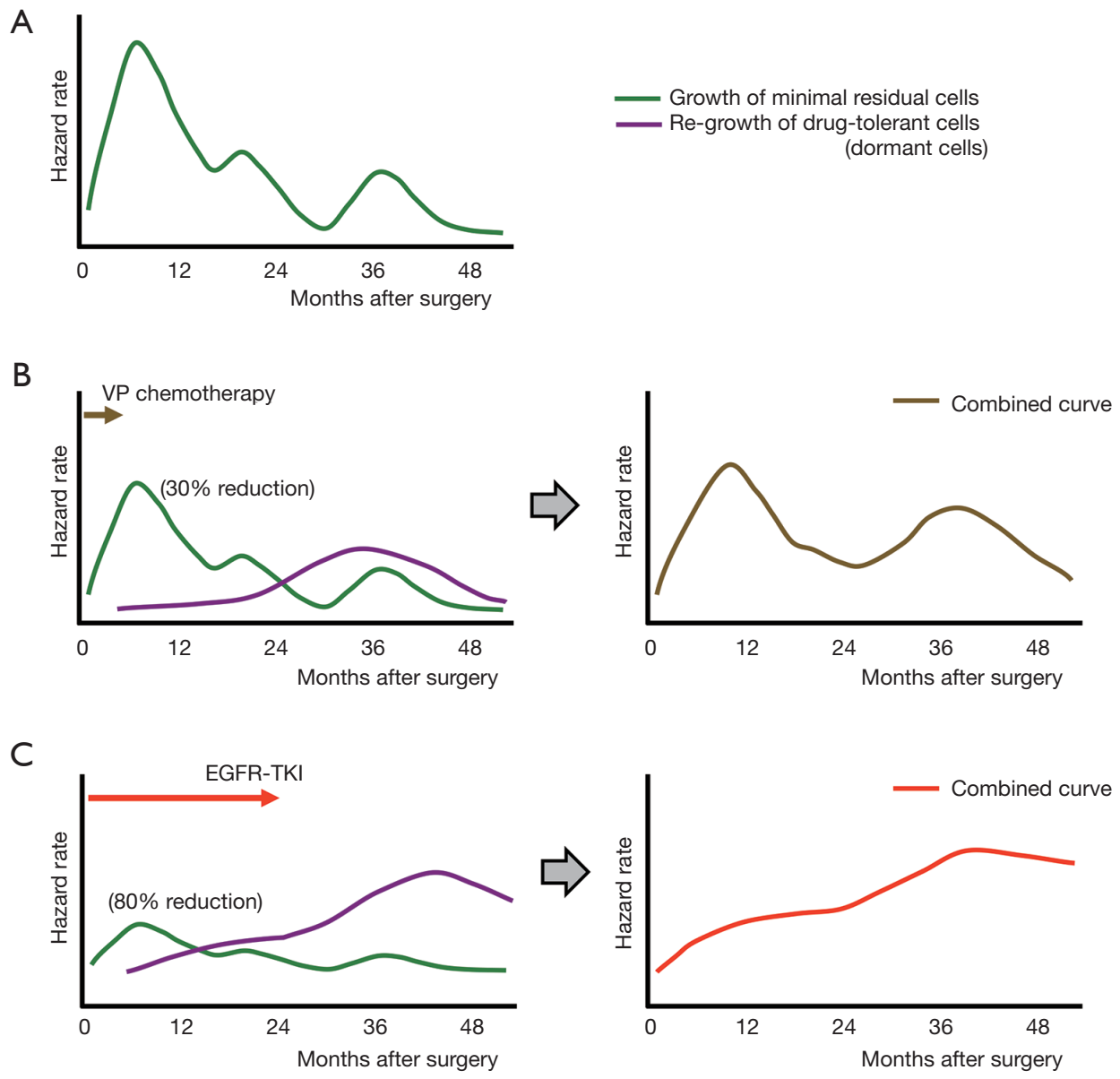

Figure 1 Estimated curves for hazard rate of treatment failure in patients who have undergone surgical resection of lung cancer. (A) Hazard curve in patients who did not receive adjuvant therapy. The curve is original which was generated by the author referring to previous studies $(14,15)$; (B) hazard curve in patients who received adjuvant VP chemotherapy; (C) hazard curve in patients who received adjuvant gefitinib monotherapy. VP, vinorelbine plus cisplatin; EGFR, epidermal growth factor receptor; TKI, tyrosine kinase inhibitor.

(Figure 1B, left). Such regrowth of drug-tolerant cells is also considered problematic in patients undergoing EGFR-TKI monotherapy for advanced disease, and several strategies to co-target drug-tolerant cells have been suggested $(12,22)$. Briefly, for example, AXL, insulin-like growth factor 1 receptor, NF- $\mathrm{B}, \beta$-catenin, STAT3, ETS-1, MCL-1, and most recently, Aurora kinase A (AURKA), have been reported as candidate molecules for co-targeting strategies to eliminate drug-tolerant cells. Furthermore, the author considers that targeting drug-tolerant cells will be more successful in the adjuvant setting than in the advanced disease setting. In a patient with advanced-stage NSCLC, we can say that the treatment will start with $1 \times 10^{11}$ cancer cells, and $3 \times 10^{8}$ drug-tolerant cells will still remain even if an EGFR-TKI eliminates cancer cells as effectively as in cell culture dishes (killing 99.7\% of cells) (12). In contrast, a surgical candidate has fewer cancer cells (e.g., $1 \times 10^{10}$ ), and "curative" resection will remove more than $99.9 \%$ of cancer cells. Therefore, if an adjuvant EGFR-TKI works as effectively as in cell culture dishes, only $3 \times 10^{4}$ drugtolerant cells will remain; therefore, a co-targeting strategy [e.g., using an AURK inhibitor (22)] will work better in the adjuvant setting than in the advanced disease setting. Third, patient selection should be optimized. Although the EVAN trial and the ADJUVANT trial demonstrated lower incidences of severe adverse effects in patients treated by EGFR-TKI than VP chemotherapy, EGFRTKI combination therapy (e.g., with an AURK inhibitor) will have higher toxicity than EGFR-TKI monotherapy. Therefore, biomarker selection, such as the use of 
circulating tumor DNA analysis (23), may be important to identify the subgroup of patients with a higher risk of recurrence.

Patients with EGFR mutation-positive lung cancer are good candidates for EGFR-TKI treatment. Compared with some patients with $E G F R$-negative NSCLC, however, these patients with $E G F R$-mutated lung cancer usually receive less benefit from cancer immunotherapy (24); such immunotherapy may provide durable and long-term efficacy as well as hope for a cure in some patients without EGFR mutation. Therefore, to increase the cure rate of patients with $E G F R$-mutated lung cancer, eliminating all cancer cells after "curative" pulmonary resection by a reasonable adjuvant therapy should be a promising strategy. Based on the post hoc analysis of the ADJUVANT trial, the author believes that the answer lies in the combination of a BBBpenetrating EGFR-TKI (e.g., AZD3759 or osimertinib) plus an agent that can effectively eliminate drug-tolerant cells [such as an AURK inhibitor (22)] for selected patients (e.g., selected by post-surgical circulating tumor DNA analysis).

\section{Acknowledgments}

Funding: This work was supported by a Grant-in-Aid for Scientific Research from the Japan Society for the Promotion of Science (grant number 18K07336 to Kenichi Suda) and by a grant from the Uehara Memorial Foundation to Kenichi Suda. The author thanks Angela Morben, DVM, ELS, from Edanz Group (www.edanzediting.com/ac), for editing a draft of this manuscript.

\section{Footnote}

Conflicts of Interest: The author has received honoraria from Boehringer Ingelheim, has served as an advisory board member for AstraZeneca, and has received research funding (through Kindai University Faculty of Medicine) from Boehringer Ingelheim and Rain Therapeutics.

Ethical Statement: The author is accountable for all aspects of the work in ensuring that questions related to the accuracy or integrity of any part of the work are appropriately investigated and resolved.

\section{References}

1. Okami J, Shintani Y, Okumura M, et al. Demographics, safety and quality, and prognostic information in both the seventh and eighth editions of the TNM classification in 18,973 surgical cases of the Japanese Joint Committee of Lung Cancer Registry Database in 2010. J Thorac Oncol 2019;14:212-22.

2. Suzuki K, Saji H, Aokage K, et al. Comparison of pulmonary segmentectomy and lobectomy: safety results of a randomized trial. J Thorac Cardiovasc Surg 2019;158:895-907.

3. Nelson DB, Mehran RJ, Mitchell KG, et al. Roboticassisted lobectomy for non-small cell lung cancer: a comprehensive institutional experience. Ann Thorac Surg 2019;108:370-6.

4. Bertolaccini L, Batirel H, Brunelli A, et al. Uniportal video-assisted thoracic surgery lobectomy: a consensus report from the Uniportal VATS Interest Group (UVIG) of the European Society of Thoracic Surgeons (ESTS). Eur J Cardiothorac Surg 2019;56:224-9.

5. Kenmotsu H, Ohde Y, Wakuda K, et al. Survival data for postoperative adjuvant chemotherapy comprising cisplatin plus vinorelbine after complete resection of nonsmall cell lung cancer. Cancer Chemother Pharmacol 2017;80:609-14.

6. Pignon JP, Tribodet H, Scagliotti GV, et al. Lung adjuvant cisplatin evaluation: a pooled analysis by the LACE Collaborative Group. J Clin Oncol 2008;26:3552-9.

7. Hirsch FR, Suda K, Wiens J, et al. New and emerging targeted treatments in advanced non-small-cell lung cancer. Lancet 2016;388:1012-24.

8. Osarogiagbon RU, Veronesi G, Fang W, et al. Early-stage NSCLC: advances in thoracic oncology 2018. J Thorac Oncol 2019;14:968-78.

9. Yue $\mathrm{D}, \mathrm{Xu} \mathrm{S}$, Wang Q, et al. Erlotinib versus vinorelbine plus cisplatin as adjuvant therapy in Chinese patients with stage IIIA EGFR mutation-positive non-small-cell lung cancer (EVAN): a randomised, open-label, phase 2 trial. Lancet Respir Med 2018;6:863-73.

10. Zhong WZ, Wang Q, Mao WM, et al. Gefitinib versus vinorelbine plus cisplatin as adjuvant treatment for stage II-IIIA (N1-N2) EGFR-mutant NSCLC (ADJUVANT/ CTONG1104): a randomised, open-label, phase 3 study. Lancet Oncol 2018;19:139-48.

11. Kelly K, Altorki NK, Eberhardt WE, et al. Adjuvant erlotinib versus placebo in patients with stage IB-IIIA nonsmall-cell lung cancer (RADIANT): a randomized, doubleblind, phase III trial. J Clin Oncol 2015;33:4007-14.

12. Suda K, Bunn PA Jr, Rivard CJ, et al. Primary doublestrike therapy for cancers to overcome EGFR kinase 
inhibitor resistance: proposal from the bench. J Thorac Oncol 2017;12:27-35.

13. Xu ST, Xi JJ, Zhong WZ, et al. The unique spatialtemporal treatment failure patterns of adjuvant gefitinib therapy: a post hoc analysis of the ADJUVANT trial (CTONG 1104). J Thorac Oncol 2019;14:503-12.

14. Demicheli R, Fornili M, Ambrogi F, et al. Recurrence dynamics for non-small-cell lung cancer: effect of surgery on the development of metastases. J Thorac Oncol 2012;7:723-30.

15. Park BJ, Cho JH, Lee JH, et al. Temporal and regional distribution of initial recurrence site in completely resected N1-stage II lung adenocarcinoma: the effect of postoperative adjuvant chemotherapy. Lung Cancer 2018;117:7-13.

16. Baxevanos P, Mountzios G. Novel chemotherapy regimens for advanced lung cancer: have we reached a plateau? Ann Transl Med 2018;6:139.

17. Mitsudomi T, Morita S, Yatabe Y, et al. Gefitinib versus cisplatin plus docetaxel in patients with non-small-cell lung cancer harbouring mutations of the epidermal growth factor receptor (WJTOG3405): an open label, randomised phase 3 trial. Lancet Oncol 2010;11:121-8.

18. Maemondo M, Inoue A, Kobayashi K, et al. Gefitinib or chemotherapy for non-small-cell lung cancer with mutated
EGFR. N Engl J Med 2010;362:2380-8.

19. Liao L, Ji X, Ge M, et al. Characterization of genetic alterations in brain metastases from non-small cell lung cancer. FEBS Open Bio 2018;8:1544-52.

20. Ahn MJ, Kim DW, Cho BC, et al. Activity and safety of AZD3759 in EGFR-mutant non-small-cell lung cancer with CNS metastases (BLOOM): a phase 1, open-label, dose-escalation and dose-expansion study. Lancet Respir Med 2017;5:891-902.

21. Reungwetwattana T, Nakagawa K, Cho BC, et al. CNS response to osimertinib versus standard epidermal growth factor receptor tyrosine kinase inhibitors in patients with untreated EGFR-mutated advanced non-small-cell lung cancer. J Clin Oncol 2018:JCO2018783118. [Epub ahead of print].

22. Suda K. Targeting the reversible drug-tolerant state: aurora kinase A, is that the final answer? Transl Cancer Res 2019. [Epub ahead of print].

23. Liang H, Huang J, Wang B, et al. The role of liquid biopsy in predicting post-operative recurrence of non-small cell lung cancer. J Thorac Dis 2018;10:S838-45.

24. Lee CK, Man J, Lord S, et al. Checkpoint inhibitors in metastatic EGFR-mutated non-small cell lung cancer-a meta-analysis. J Thorac Oncol 2017;12:403-7.
Cite this article as: Suda K. For a better adjuvant strategy for resected lung cancer-lessons from treatment failure patterns of the ADJUVANT trial (CTONG 1104). Transl Lung Cancer Res 2019;8(Suppl 4):S395-S399. doi: 10.21037/tlcr.2019.08.24 